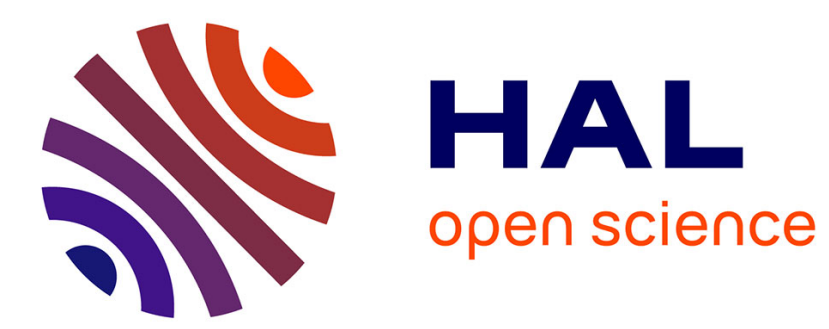

\title{
Coupling Transformer Operation of a Dynamic Voltage Restorer Under Electrical Grid Conditions
}

Virginie Majchrzak

\section{To cite this version:}

Virginie Majchrzak. Coupling Transformer Operation of a Dynamic Voltage Restorer Under Electrical Grid Conditions. 2017. hal-01536606v1

\section{HAL Id: hal-01536606 https://hal.science/hal-01536606v1}

Preprint submitted on 11 Jun 2017 (v1), last revised 21 Jun 2017 (v2)

HAL is a multi-disciplinary open access archive for the deposit and dissemination of scientific research documents, whether they are published or not. The documents may come from teaching and research institutions in France or abroad, or from public or private research centers.
L'archive ouverte pluridisciplinaire HAL, est destinée au dépôt et à la diffusion de documents scientifiques de niveau recherche, publiés ou non, émanant des établissements d'enseignement et de recherche français ou étrangers, des laboratoires publics ou privés. 


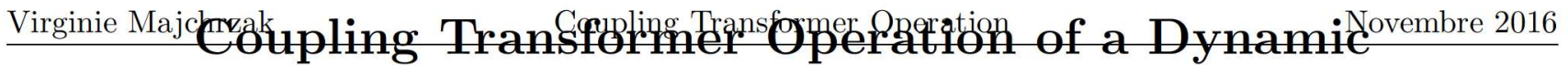 Voltage Restorer Under Electrical Grid Conditions
}

\author{
Virginie Majchrzak \\ Version provisoire - Novembre 2016
}

\begin{abstract}
ABSTR.ACT
To improve electrical grids power quality, a dynamic voltage restorer with a magnetic voltage source converter protection scheme integrated in the coupling transformer is developed. Finite element analyses with an electricalmagnetic coupling modeling are performed to study the behavior of the coupling transformer for all the operation modes, both the voltage regulation one in normal operation and the voltage source converter protection mode for several different and harsher electrical grid fault conditions in fault operation.
\end{abstract}

Keywords - Power system modeling, short-circuit currents, transformer cores, voltage fluctuations.

\section{INTRODUCTION}

Voltage fluctuations of electrical grids are a major problem, liable for power quality decreases ${ }^{1}$ and economic losses. ${ }^{2}$ Among all existing Flexible AC Transmission Systems (FACTS) and custom power devices

This device is composed of a coupling transformer whose first winding is inserted in series in the electrical grid and second one is connected to an energy storage unit through a Voltage Source Converter (VSC). However, as it is inserted in the electrical grid continuously, the protection of the VSC against high fault currents constitutes its weak point. So, two solutions are usually used for the existing DVR, to avoid damaging the VSC. The first one consists in oversizing it, but it is expensive and it increases the size of the converter. ${ }^{3}$ The second one consists in inserting an electro-mechanical bypass in parallel with the coupling transformer to disconnect it from the electrical grid during fault periods, but the time response of this protection scheme is quite high, about $100 \mathrm{~ms}^{4,5}$

To improve the protection of the VSC, the proposed solution is based on a magnetic bypass integrated inside the magnetic core of the coupling transformer. ${ }^{6}$ It is realized by the adding of a return leg to a standard transformer and the use of the virtual air gap principle ${ }^{7}$ to modify the distribution of the magnetic flux according to the electrical grid conditions.

Up to now, the authors studied the operating principle, in Fault operation, of this device with a single phase reduced-scale device. ${ }^{8}$ The capacity of the magnetic bypass to limit the current in the winding connected to the VSC was validated by both Finite Element Analysis (FEA) and laboratory experiments. Then, based on this result, the authors extrapolated the operation of such a device at industrial rated electrical values. The design of the $1 \mathrm{kVA}$-prototype was realized with FEA and the operation of the return leg as a magnetic bypass was successfully validated with experiments in Fault operation up to three times the rated current. ${ }^{9}$ The aim of this paper is twofold. Firstly, validate that the original function of a DVR, i.e operating as a voltage transformer in Normal operation, is not impacted by the addition of the return leg. This is done both by experiments and FEA. Secondly, based on the promising results previously experimentally obtained, ${ }^{9}$ FEA are performed to study the performances of the VSC protection scheme during very critical electrical grid fault current conditions for which safety and reproducible experiments can not be done. Firstly, the authors describe the architecture of the device and explain how they study its operation. Then, they analyze the performances of the coupling transformer for each aforementioned operation mode. 


\section{DYNAMIC VOLTAGE RESTORER WITH THE VSC PROTECTED BY A MAGNETIC BYPASS}

\subsection{Coupling transformer with a magnetic bypass}

The magnetic core of the coupling transformer (Fig. 1) ${ }^{9}$ of the DVR whose VSC protected by a magnetic bypass is composed of two parts: a classical transformer part and a return leg. ${ }^{6}$ The transformer part is the exact same as those of existing DVRs. The first winding is inserted in series in the electrical grid between a power supply and critical loads. The second winding is connected to an energy storage unit through a VSC to inject the appropriate voltage in case of an upstream clectrical grid voltage fluctuation. The return leg, composed of four pairs of holes, aims to protect the VSC by deflecting the magnetic flux generated in the transformer part, from the leg connected to the VSC to the return leg, in Fault operation. This magnetic bypass is based on the virtual air gap principle, ${ }^{7}$ i.e the local saturation of a magnetic core by auxiliary windings inserted in drilled holes and supplied by a DC current. Table 1 gives the characteristics of this device.

Table 1. Characteristics of the low voltage coupling transformer

\begin{tabular}{ll}
\hline \hline Rated AC Voltage $V_{\mathrm{sn}}$ & 23 Vrms \\
Rated AC Current $I_{\mathrm{pn}}$ & $41 \mathrm{Arms}$ \\
Rated Power & $1 \mathrm{kVA}$ \\
Size of the magnetic circuit & $30 \mathrm{~cm} \times 30 \mathrm{~cm} \times 4.8 \mathrm{~cm}$ \\
(Length $\times$ Width $\times$ Thickness) & \\
Magnetic sheets & PowerCore CGO135-35 \\
Number of turns of the main windings & 32 \\
Number of turns of the auxiliaries windings & $4 \times 80$ \\
\hline \hline
\end{tabular}

\subsection{Test protocol}

Experiments have been made in laboratory (Fig. 2) to validate each operation modes of the prototype, only for specific electrical grids conditions. In Normal operation, the primary winding is connected to an AC sinusoidal voltage source $\left(V_{\text {Supply,rated }}=230 \mathrm{~V}\right)$ and a resistance which corresponds to the upstream and the downstream electrical grid respectively. The secondary winding is, as for it self, short-circuited for the steadystate operation mode and connected to an $\mathrm{AC}$ voltage source $\left(V_{\mathrm{s} \text {,rated }}=0.1 V_{\text {Supply,rated }}=23 \mathrm{~V}\right)$ for the voltage regulation operation mode. For the Fault operation, a high current is generated in the primary winding during

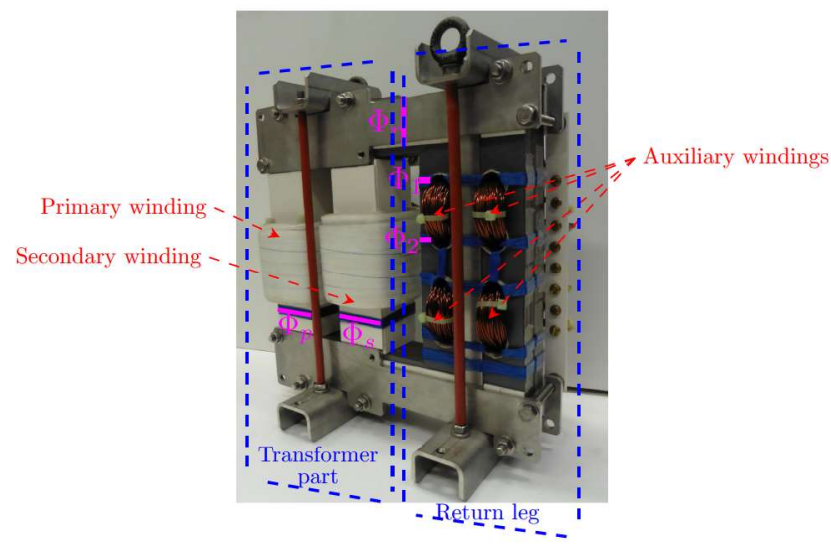

Figure 1. Experimental device of the coupling transformer 
the starting of a $22 \mathrm{~kW}$-induction machine. For all these operation modes, the auxiliary windings are connected in series to a voltage DC source and are supplied by a DC current $\left(I_{\text {Aux,rated }}=5 \mathrm{~A}\right)$.

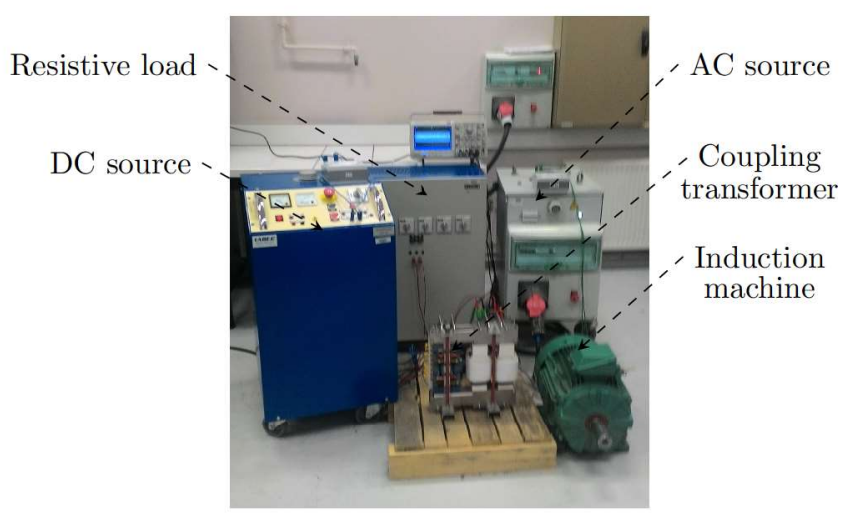

Figure 2. Test bed with the coupling transformer

\subsection{Finite Element modeling}

Since the operation of the coupling transformer combines both DC and AC magnetic flux, the magnetic state of the return leg depends on a non-linear relation including both currents $i_{\mathrm{p}}$ and $i_{\text {Aux. }}{ }^{8}$ Thus, analytic methods can not describe all the operation of the device. Besides, analyzing its operation during harsh grid fault conditions can not be done by experiments because of safety and reproducibility of the tests. For all these reasons, the behavior of the device is studied with FEA. A 2D-modeling has been performed by using the magnetic vector potential formulation. The non-linearity of the magnetic steel is taken into account by using first magnetization $\mathrm{B}(\mathrm{H})$ curves. As for the electrical circuits, the windings are excited by using electrical circuit equations coupled to the FE equations in order to connect them either to a voltage source or to a current source according to the operation of the coupling transformer. This modeling allows to analyze the magnetic flux distribution, the current and the voltage of the windings and the sources for all these operation modes and for all electrical grid conditions.

\section{NORMAL OPERATION}

In Normal operation, the return leakage leg is saturated and the device acts like a real voltage transformer (Fig. 3). ${ }^{8}$ Thus, in the voltage regulation operation mode, it is able to compensate electrical grid voltage fluctuations.

FEA allow to study the influence of the amplitude of the voltage at the secondary windings terminals, on the performances of the coupling transformer during the voltage regulation operation mode. The injected voltage at the secondary winding terminals is the difference between the rated voltage of the power supply and its value during the dip. Fig. 4 shows the variation of the ratio between the voltage of the transformer during this operation mode. It shows it does not depend so much of the injected voltage $v_{\mathrm{s}}$. Besides, it highlights that the DC current allows the coupling transformer to act as an efficient voltage transformer and so, the voltage ratio is increased by $i_{\text {Aux }}$ up to almost one.

Fig. 5 describes the experimental results for the voltage regulation operation mode with the voltage value at the secondary windings terminals $V_{\mathrm{s}}$ is equal to $0.1 V_{\text {Supply. }}$. Indeed, in case of a voltage fluctuation 


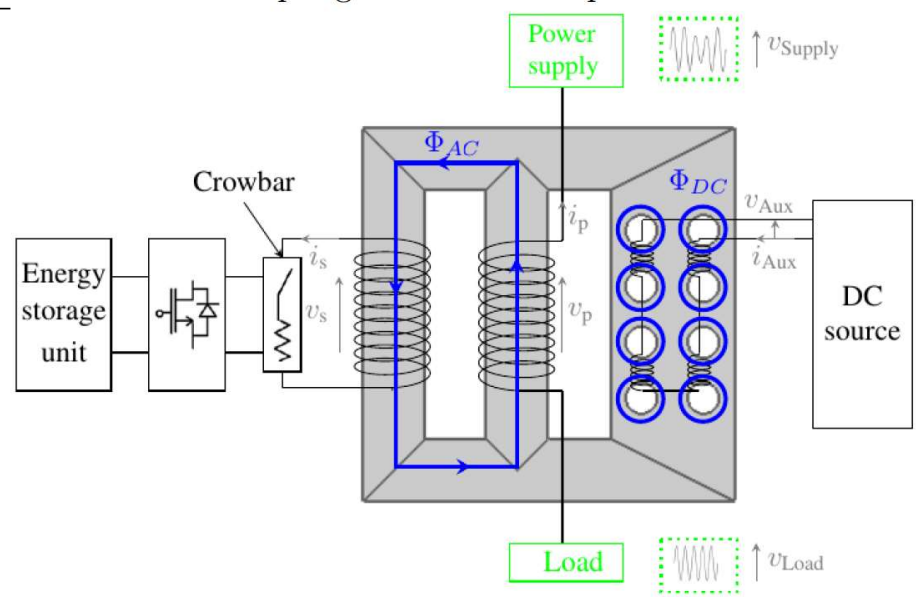

Figure 3. Normal operation of the coupling transformer

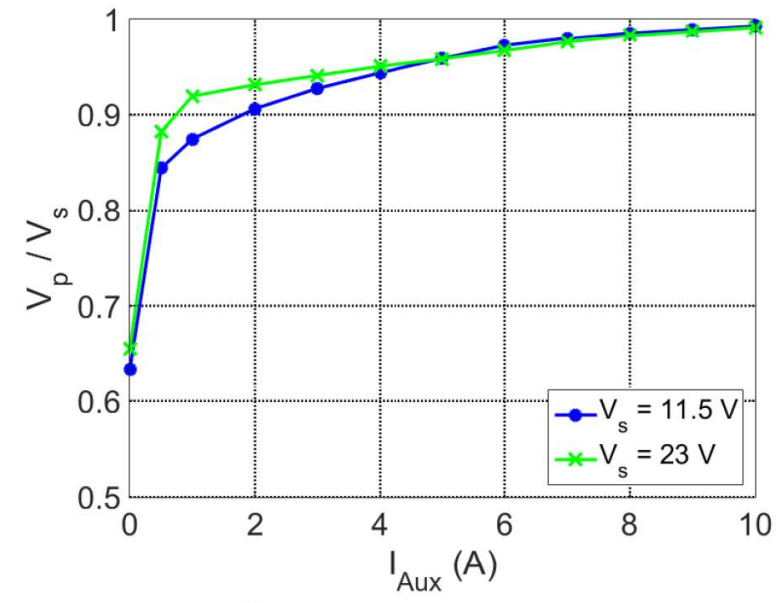

Figure 4. Voltage ratio $\frac{V_{\mathrm{P}} \max }{V_{\mathrm{s}} \max }$ according to $I_{\mathrm{Aux}}$ in Normal operation

in the electrical grid, i.e, a difference of voltage between the powcr supply tcrminals $v_{\text {Supply }}$ and the load terminals $v_{\text {Load }}$ (Fig. 5a and Fig. 5b), the DVR is able to inject the appropriate voltage $v_{\mathrm{s}}$ (Fig. 5c). Moreover, as concerns the voltage at the primary winding, $V_{\mathrm{p}, \max }=0.99 V_{\mathrm{s}, \max }$ (Fig. 5c), that means the coupling transformer acts well as a voltage regulator when the return leg is saturated. Consequently, the voltage $v_{\text {Load }}$ is equal to the sum of $v_{\mathrm{s}}$ and $v_{\text {Supply }}$. Therefore, $v_{\text {Load }}$ does not vary in case of power supply voltage variations (Fig. $5 \mathrm{~d}$ ). Besides, the local saturation of the return leg effects are limited in Normal operation since the difference of the THD between the power supply and the load is equal to $0.4 \%$.

Therefore, in Normal operation, FEA and experimental results shown that the coupling with a magnetic bypass operates as well as it was expected, in accordance with the operating principle of such a device.

\section{FAULT OPERATION}

During the Fault operation, the AC magnetic flux, mostly generated by the fault current in the primary winding, is higher than the DC magnetic flux. Hence, it circulates in the return leg instead of the leg connected to the VSC (Fig. 6). So, in Fault operation, the deflection of the AC magnetic flux in the return leg allows the magnetic bypass to act as a VSC electrical grid fault current protection scheme. 


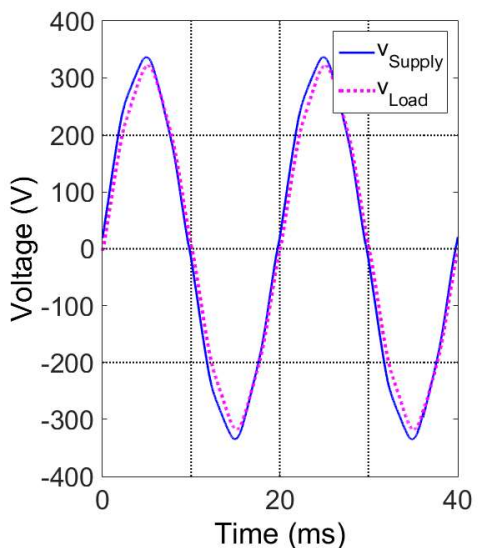

(a)

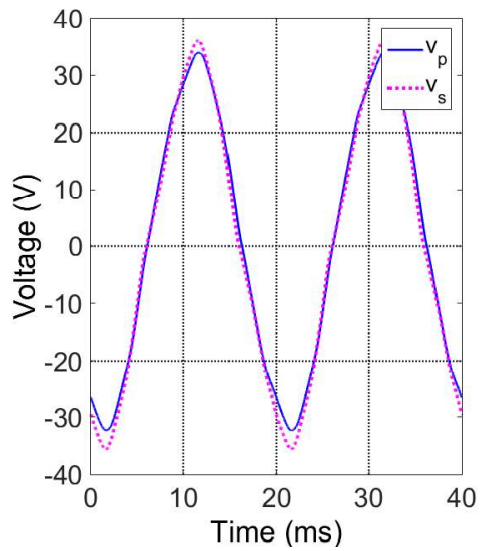

(c)

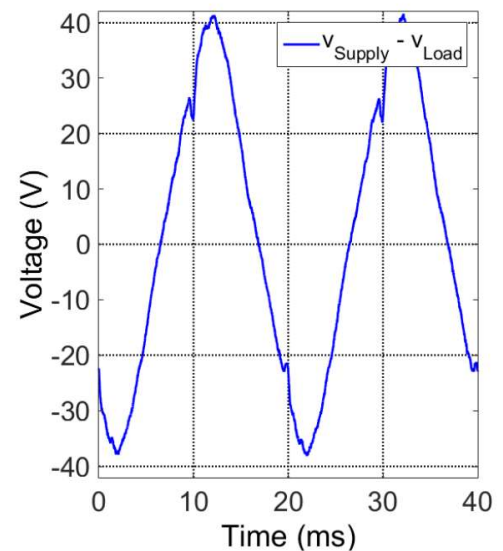

(b)

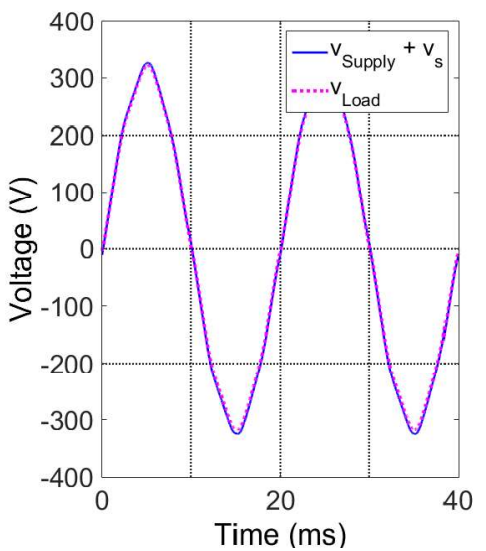

(d)

Figure 5. Experiments during the voltage regulation operation mode: $V_{\mathrm{s}, \mathrm{rms}}=23 \mathrm{~V}, V_{\mathrm{Supply}, \mathrm{rms}}=230 \mathrm{~V}$ and $I_{\mathrm{Aux}}=5 \mathrm{~A}$

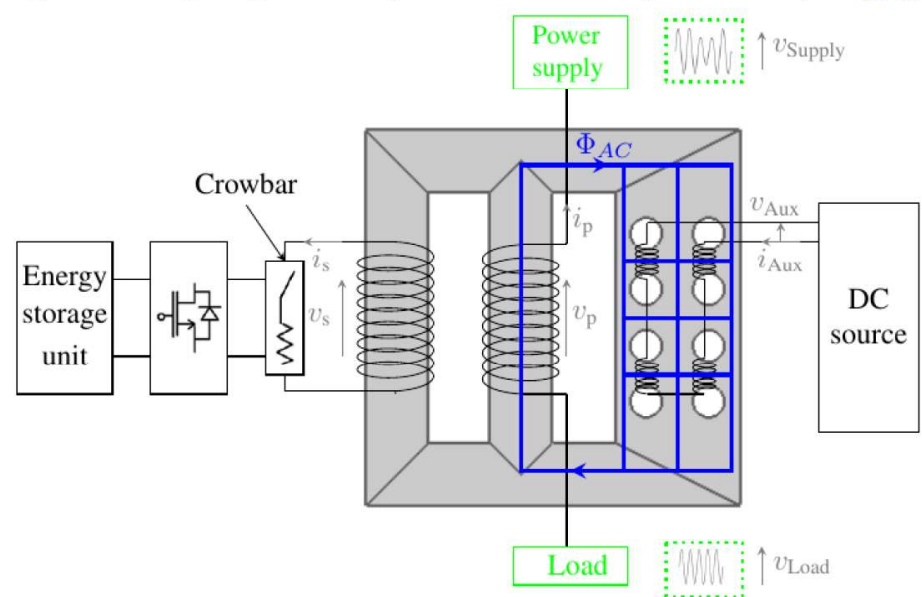

Figure 6. Fault operation of the coupling transformer

In this operation mode, two strategies exist to supply the auxiliary windings: keep $i_{\mathrm{Aux}}$ at the same value than in Normal operation or cancel $i_{\text {Aux }}$. Fig. 7 describes the influence of the currents $i_{\mathrm{p}}$ and $i_{\mathrm{Aux}}$ on the limitation of the current $i_{\mathrm{s}}$, for both strategies. It shows that the latter is better when $i_{\text {Aux }}$ is equal to zero, that it was expected. 


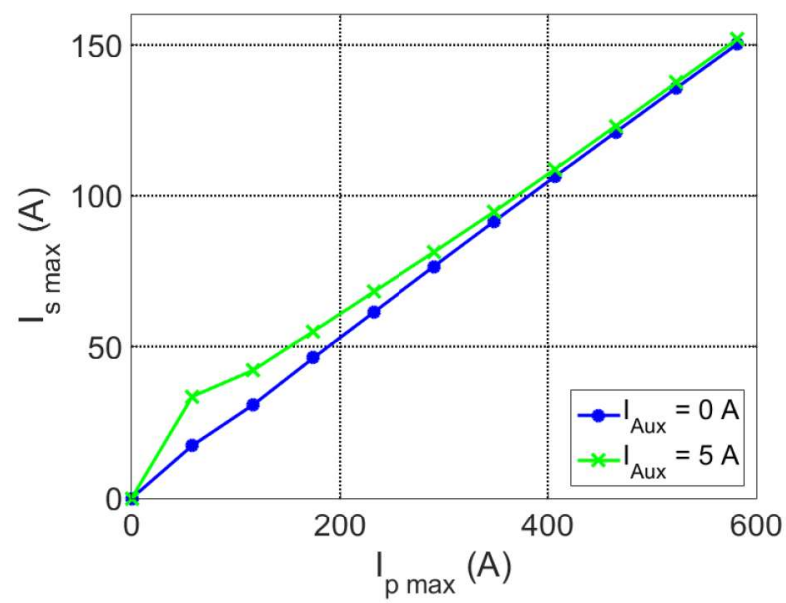

Figure 7. Variation of $i_{\mathrm{s}}$ according to $i_{\mathrm{p}}$ and $i_{\mathrm{Aux}}$

Besides, for very high fault current $\left(I_{\mathrm{p}}=6 I_{\mathrm{p}, \text { rated }}\right.$ in Fig. 8), the current $i_{\mathrm{s}}$ is still limited which also validates the operation of the coupling transformer under extreme electrical grid fault conditions, as concerns the electrical aspect. This is explained by two phenomena: first the desaturation of the return leg by the circulation of the $\mathrm{AC}$ magnetic flux in the latter, and then, the saturation of the primary leg.

The second parameter studied with FEA is the influence of the fault occurence instant. Fig. 9 describes the behavior of the coupling transformer when a fault appears at an extremum of the current $i_{\mathrm{p}}$. There is not any difference as concerns the limitation of $i_{\mathrm{s}}$ between Fig. 8 and Fig. 9. Consequently, the fault instant does not modify the behavior of the device, only the intensity of the fault current plays a role.

As concerns the crowbar, up to now, it has been assumed to be in closed position in Fault operation and so, the secondary winding short-circuited. Experiments described in Fig.10a shows the capability of the coupling transformer to limit the current in the winding connected to the VSC, in this case $I_{\mathrm{s}, \max }=24 \% I_{\mathrm{p}, \max }$.

Nevertheless, close and open a crowbar requires to detect electrical grid faults. That is why, the performances of the coupling transformer without closing the crowbar during faults are studied. Fig. 10b shows the variation of the currents and the voltage during the motor starting with the crowbar open. Compared to the previous results, let the crowbar open increases the current $i_{\mathrm{s}}\left(I_{\mathrm{s}, \max }=37 \% I_{\mathrm{p}, \max }\right)$ but it is already limited. Hence, the closure of the crowbar allows a better limitation of the current but needs a detection system.

Therefore, in Fault operation, FEA and experimental results shown that the prototype operates as efficiently as the operating principle mentioned.

\section{CONCLUSION}

To conclude, the authors validated each operation modes of the coupling transformer with a magnetic bypass of a DVR, considering the electrical aspect only. The operation has been studied with both experiments for only specific electrical conditions and with FEA for the extreme electrical grid conditions. It has been shown that the device is able to:

- to behave as a real voltage transformer in Normal operation and to compensate electrical grid fluctuations, with a voltage ratio almost equal to one, 

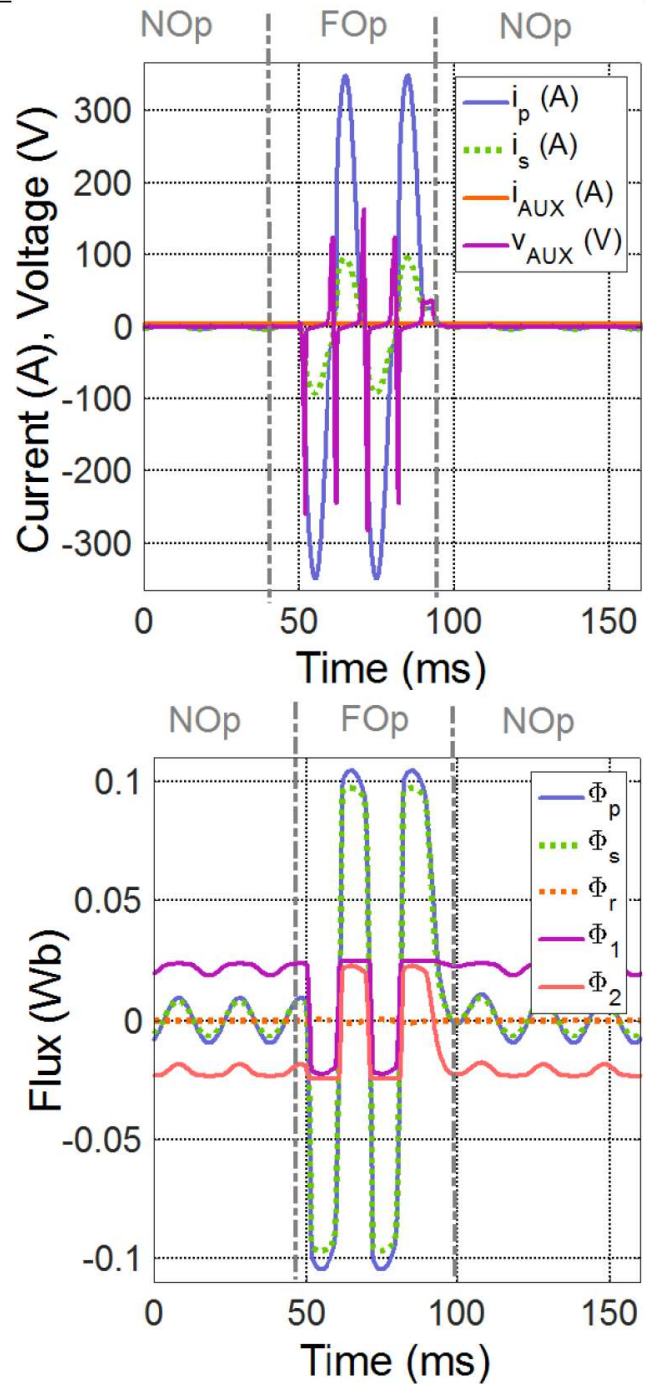

Figure 8. Behavior of the coupling transformer during a high grid fault current

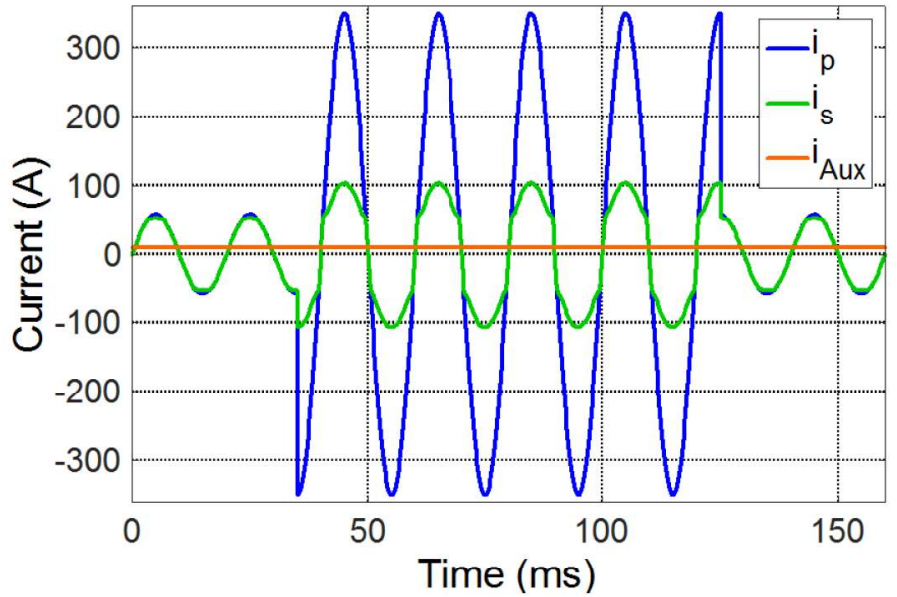

Figure 9. Behavior of the coupling transformer during a high grid fault current which appears at the extremum of $i_{\mathrm{p}}$ 


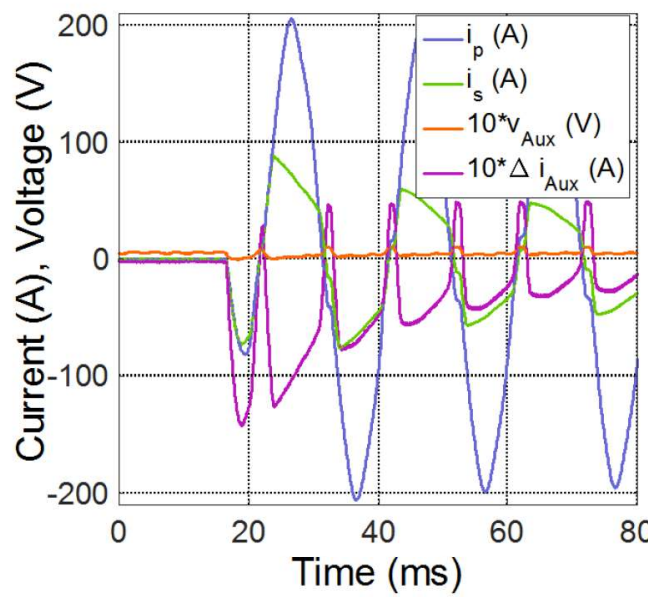

(a) $V_{\mathrm{s}}=0 \mathrm{~V}$

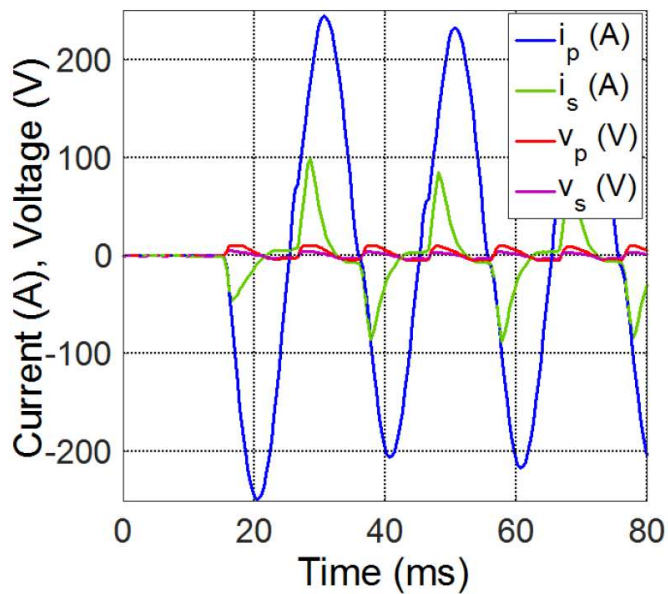

(b) $V_{\mathrm{s}}=11.5 \mathrm{~V}$

Figure 10. Starting of the motor with with $I_{\mathrm{Aux}}=5 \mathrm{~A}$

- to limit the current in the VSC for very high electrical grid fault current, whatever the fault current waveform and until six times the rated electrical grid current for the considered FEA.

These results highlight that the designed coupling transformer operates efficiently as a voltage regulator as well as a fault current VSC protection scheme. Future works will use these results to add the control of VSC and manufacture a $20 \mathrm{kV}$-device for medium voltage electrical grid.

\section{REFERENCES}

1. N. Edomah, "Effects of voltage sags, swell and other disturbances on electrical equipment and their economic implications," in Electricity Distribution - Part 1, 2009. CIRED 2009. 20th Int. Conf. and Exhibition on, Jun. 2009, pp. 1-4.

2. Z. Klaić, D. Sipl, and S. Nikolovski, "Economic impact of power quality disturbances," in 222nd Int. Conf. \& Exhibition on Electricity Distribution, Electricity Distribution Systems for a Sustainable Future, Jun. 2013.

3. T. Jimichi, S. Member, H. Fujita, and H. Akagi, "Design and experimentation of a dynamic voltage restorer capable of significantly reducing an energy-storage element," IEEE Trans. Ind. Appl., vol. 44, no. 3, pp. 817-825, May 2008.

4. N. Woodley, L. Morgan, and A. Sundaram, "Experience with an inverter-based dynamic voltage restorer," IEEE Trans. Power Delivery, vol. 14, no. 3, pp. 1181-1186, Jul. 1999.

5. J. Magnusson, J. A. Martinez-Velasco, A. Bissal, G. Engdahl, and L. Liljestrand, "Optimal design of a medium voltage hybrid fault current limiter," in Energy Conf. (ENERGYCON), 2014 IEEE Int., May 2014, pp. $431-438$.

6. P. Guuinic, J.-F. Brudny, V. Costan, and M. Dessoude, "Series voltage regulator with electronics protected against short-circuits by magnetic circuit-based decoupling using holes and windows," 2013.

7. V. Molcrette, J.-L. Kotny, J.-P. Swan, and J.-F. Brudny, "Reduction of inrush current in single-phase transformer using virtual air gap technique," IEEE Trans. Magn., vol. 34, no. 4, pp. 1192-1194, Jul. 1998.

8. V. Majchrzak, G. Parent, J.-F. Brudny, V. Costan, and P. Guuinic, "Coupling transformer with a virtual air gap for the protection of dynamic voltage restorers," in IECON 2014 - 40th Annu. Conf. of the IEEE Ind. Electron. Soc., Oct. 2014, pp. 462-468.

9. — - "Design of a coupling transformer with a virtual air gap for dynamic voltage restorers," IEEE Trans. Magn., vol. 52, no. 7, pp. 1-4, Jul. 2016. 